\title{
EFFICIENCY AND ACCURACY OF SIMULATED MICROSTRUCTURE IMAGES GENERATED BY MACHINE LEARNING
}

\author{
GĄDEK-MOSZCZAK A. ${ }^{1}$, FILIPOWSKA R. ${ }^{2}$ \\ ${ }^{1}$ Cracow University of Technology \\ Faculty of Mechanical Engineering \\ aneta.gadek-moszczak@pk.edu.pl \\ ${ }^{2}$ Cracow University of Technology \\ Faculty of Mechanical Engineering \\ Renata.filipowska@pk.edu.pl
}

Key words: material microstructure, machine learning, neural networks, simulation

\begin{abstract}
.
Computer image analysis is a well-known method in material science, mechanical engineering and other branches of science and engineering. Application of the machine learning method in image processing delivers promising results, especially in images with a high level of noise and low contrast. Employing automatic classification, predictive models, simulation models deliver a huge benefit in research. The former model of science-based mainly on experiments slowly passing away. It is still an important part of research but, using advanced computer methods save time and allow significantly reduces the cost of studies. It begins new branch of material science and new research challenges, interdisciplinary filed of materials informatics, incorporating scientists exploring, mathematics, informatics and material science.
\end{abstract}

\section{INTRODUCTION}

Nowadays advance computer methods as machine learning are widely applied in all fields of science. Employing automatic classification, predictive models, simulation models deliver a huge benefit in research. The former model of science-based mainly on experiments slowly passing away. It is still an important part of research but, using advanced computer methods save time and allow significantly reduces the cost of studies. Instantly develop of algorithms method of widely knowns machine learning field attract the attention of it benefits in numerous fields of science [1].

The tests of material properties include many physical, chemical and imaging tests that allow for the control and design of more and more sophisticated and complex materials.

Getting to know a material by discovering its structure and understanding the phenomena that allow it to shape its properties is essential for designing new materials $[2,3]$. 
Considering the parameters that are important for the design and selection of material for a specific application, it is taken into account its properties:

- mechanical (strength, plasticity/elasticity, stiffness, hardness, vibration damping, fatigue resistance, crack resistance),

- physical (density, melting point, thermal and electrical conductivity, superconductivity, semi conductivity, magnetic properties),

- chemical (resistance to the external environment)

- technological (possibility of processing, shaping elements and joining, recycling).

Knowing the operational properties of the element, we can, based on the above characteristics, choose the best available materials, thus guaranteeing a long service life of the designed structural element.

By analysing the course of the material design process, we can divide it into several basic stages, the implementation of which brings us closer to obtaining the optimal, from the point of view of the initial assumptions, materials $[4,5]$ :

I. Concept - on the basis of the assumed operating conditions and necessary functional properties, we define the expected properties of the material (physical-mechanical, chemical, magnetic).

II. Preparation of the preliminary design considering the selection of the base material (metal alloys, ceramics, polymers, composite materials), the proposed manufacturing technology and the expected microstructure.

III. Production of prototypes. Most ofter

produced in order to obtained properties.

IV. Experimental

properties, and analysis subsequent quality cont
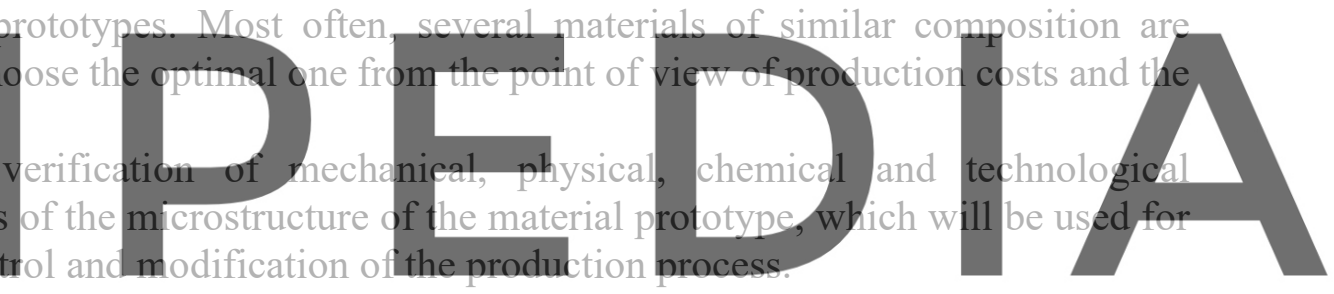

V. Statistical analysis of the results, which is used to make a de facto qualitative and not

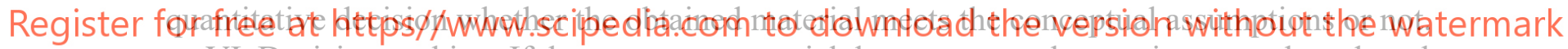

VI. Decision-making. If the prototype material does not meet the requirements, based on the analysis of the results of structural tests and properties, the composition (chemical, component content in composite materials) or the manufacturing technology is changed and it is returned to point III. Manufacturing of a prototype. If the material meets the required properties, the design cycle ends.

The design of materials assumes that two materials, produced either way, but having the same structure, will have the same properties. At the same time, two materials with identical chemical composition, but differing in structure, e.g. due to different manufacturing methods, will have different properties.

Contemporary techniques of simulating material structures, using FEM methods, simulations using machine learning techniques or Markov processes, make it possible to obtain the desired material properties by designing their structure. The classic process of creating new materials has been reversed, which has opened up new possibilities for the development of modern materials and has broken the old design path $[3,4]$. 


\section{QUANTITATIVE IMAGE ANALYSIS AND CLASSIFICATION}

Combine of big set of data and analysis with CNN allows to deliver new, hidden relations between them and results in obtaining new knowledge of analyses phenomena or material's properties. The application of advance informatic tool in material science and machine learning in particular, sets a new path in the development of material science.

According to Scopus citation database, the number of the scientific papers present the achieves in the area of application the machine learning methods is significantly increasing (fig. 1). The most active subject's area s computer science, engineering and mathematics (fig. 2), what indirectly inform about intense development of algorithmic tools, and software employed in machine learning methods. Others areas of science, like biochemistry, medicine, physic and astronomy, pharmacology and material science applied those worked out methods. The wide range of application shown very high usefulness of machine learning in scientific research regardless of the subject of analysis. Machine learning methods gives a tool to analyse the large sets of data, and highly efficient computer systems, what is an opportunity for all scientists to find and explore not obvious relationship between them. Considering the application in material science analysis of relationships between the structure composition and physical-mechanical properties gives the opportunity to design the material according the desire specification.
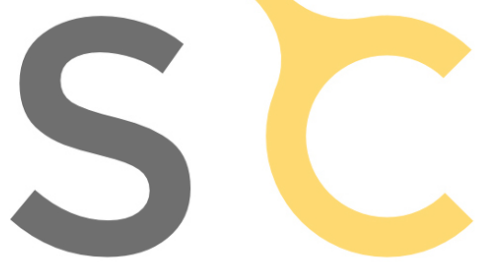

Documents by year
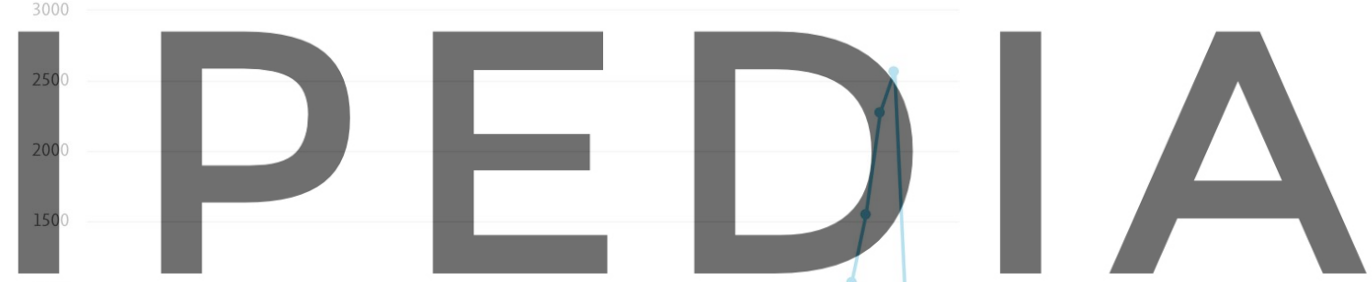

Register for free at https//www.scipedia.com to download the version without the watermark

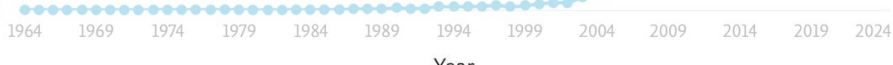

Figure 1. Number of publication per year in the subject area:

"machine learning" and "material science" according to Scopus database

Classification of the structure components on the way of standard image analysis method (fig. 2) compound the several steps: image acquisition, pre-processing due to reduce image distortion, detection of structure components, performing the quantitative analysis and conclusions based on obtained results [6].

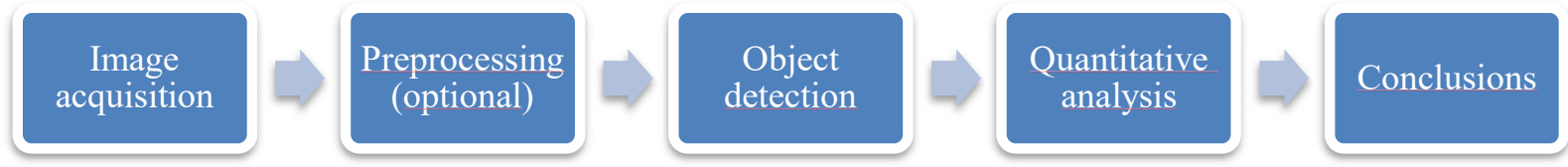

Figure 2. Scheme of the typical image analysis algorithm 
Detection procedure in this approach is based on the filtration and transformation of the images. Due to extract from the image the area that represent an object the edge detection filters, texture detection or threshold might be applied. What should be stressed out, on the level of design the automatic algorithm, the expert have full control on the results of detection, what make the algorithm bias by its subjectivity and knowledge from the very beginning, when the target of detection process is define. Stereological parameter of the structure components, its shape, fractal dimension, colour, and texture might be used in classification algorithm [6,7].

Considering the advantages of image analysis approach to object detection and classification, it is important that there are the well-established and knows procedures. Many of automatic and semi-automatic algorithms are available in commercial and open source software. Designing the automatic method for detection and classification objects may be based on small, representative set of test image.

Although to apply this method the specific knowledge from the computer vision area is needed and what is the most important expert knowledge in material science. As a disadvantage of classical approach to detection and classification structure components can be consider high impact and eventual bias the results by knowledge and experience of expert in identification the specific structure components on the stage of defining the subject of the detection, high impact of the image quality on the results - for instance the noise or uneven background. Also, the time-consuming processing in case of a large number of images.
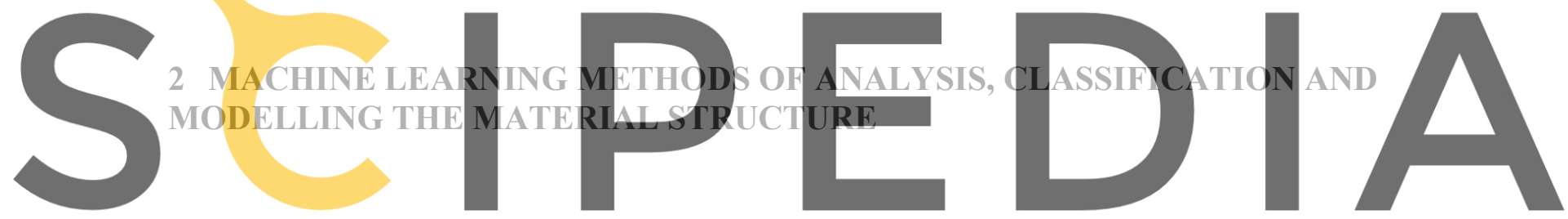

Machine learning methods are a part of artificial intelligence (AI) area. Machine learning

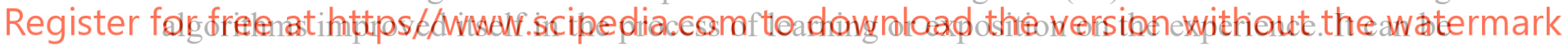
compared to natural human process of learning by experience. These algorithms create mathematical model based on the gathered data set, named training data set which deliver prognosis or decision results without direct programming each step of the processing data, how it is in convectional algorithms (fig. 3).

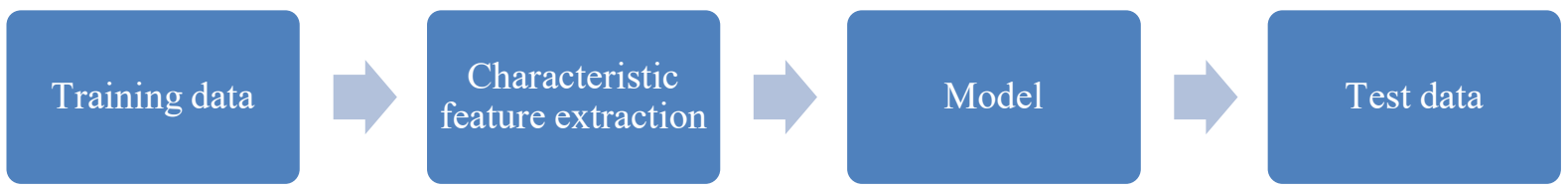

Figure 3. Model of the machine learning data processing

There are several widely apply and established algorithms used in machine learning methods $[1,3,10]$. The first group can include: weighted neighbourhood clustering that examples might be decision trees, random forest or k-Nearest neighbour, are applied. These algorithms can be applied due to assess the regression, carry out the classification, clustering and assess the similarity of structure. The results of the analysis are obtained from the intuitive relationships 
between tested images. Linear and nonlinear dimensionality reduction can be lead on the way of applying Principle Component Analysis (PCA), Support vector regression (SVR), or Nonnegative matrix factorization (NMF). These algorithms are used in experimental design, model dimensionality reduction, descriptor analysis and regression. Search algorithms like genetic algorithms (GA) or evolutionary algorithms may be applied to design the alloy or optimization of the created model. Search algorithms give promising results for material properties described geometrically, for instance, topology. The last group of the algorithm used in machine learning methods are artificial neural networks, convolutional neural networks $(\mathrm{CNN})$ and General adversarial networks (GAN). The neural network algorithm is widely applied in the classification, regression, identification and detection of objects in the images. From all machine learning, neural networks are the most popular and the most developed.

In general, we can specify the following area of application the machine learning in material science application: Classification the element of structures [9, 11-13]

- Prediction of material properties based on a set of properties - structural and mechanical $[14]$

- Structure-oriented design: design a new material, and predict their properties before conducting the physical test $[15,16]$

- Design new compounds and their structure from an input composition [16] Invers design - from required properties to the molecular structure [2, 17]

Due to obtained accurate results of the analysis the large set of data must be prepared as a training data set and if is the major difficulties in widespread application especially in the analysis of the images element the training dat research institution crea of material structures and its properties. An example of datab Cambridge Structural D
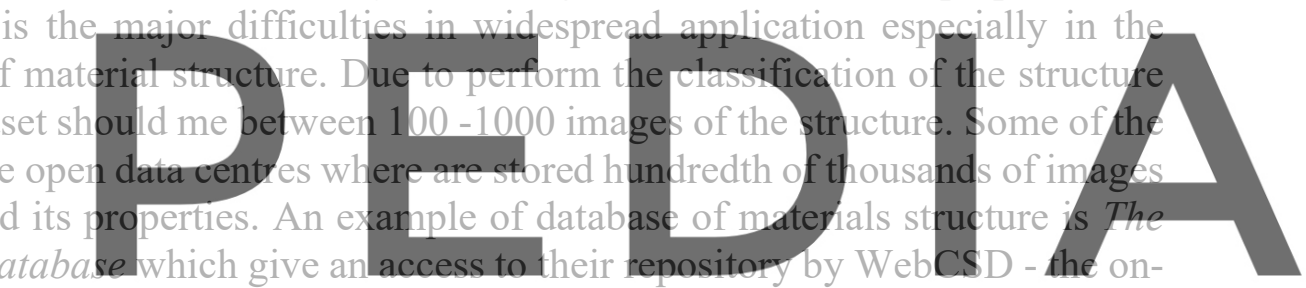

line portal. The number of deposited structures is over million. Another repositories of

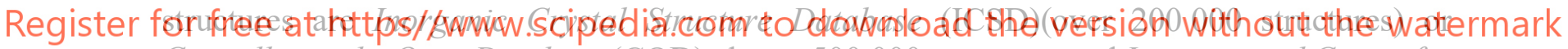
Crystallography Open Database (COD) almost 500000 structures and International Centre for Diffraction Data (ICDD) where are more then million structures. Those databases give a great opportunity to take a part in developing new application of machine learning in structure recognition and classification.

Due to create the prediction model of properties of design material the analysed data set must present structure and physical-mechanical properties. The access to those type of data is available online for instance in $A F L O W$ (Automatc Flow to material discovery) were users have access to more than 3 millions of material compounds with over 600000 calculated properties. The Materials Project and Open Quantum Materials Database (OQMD) are also the online databased which gives opportunity download the needed datasets to created adequate model. In order to process large data sets, it is also necessary to use computers with high computing power, what is also still an obstacle to wide application of machine learning methods.

\section{CONCLUSIONS}

Machine learning open up promising possibilities in material analysis and design. It is efficient tool that intensifies research on materials and, in combination with classic stereological 
techniques, will improve the analysis of large sets of image data, providing valuable data. It begins new branch of material science and new research challenges, interdisciplinary filed of materials informatics, incorporating scientists exploring, mathematics, informatics and material science. The large structure database with online access and open for researcher are an opportunity to employ the machine learning methods wider with using shared data set.

The solutions presented here can also be useful in areas where analytical approaches have been used so far, including analysis of plates [18] or beams [19]. Similar methods are adopted in other areas of science, including statistical data analysis [20], industrial process prediction [21] or semi-parametric models [22]. However, the importance of this approach for material sciences is essential, enabling the combination of experimental and simulation research (SBES - Simulation-Based Engineering and Science), which allow for an insight into phenomena with a large number of parameters and their mutual interactions, e.g. influence of nano-inclusions $[23,24]$, modification of the surface layer [25-27], corrosion phenomena [28, 29] or thermomechanical loads $[30,31]$. It can also be useful when designing the structure of specific industrial databases $[32,33]$ for images storing, as well as visual control of non-contact shaping techniques $[34,35]$

\section{REFERENCES}

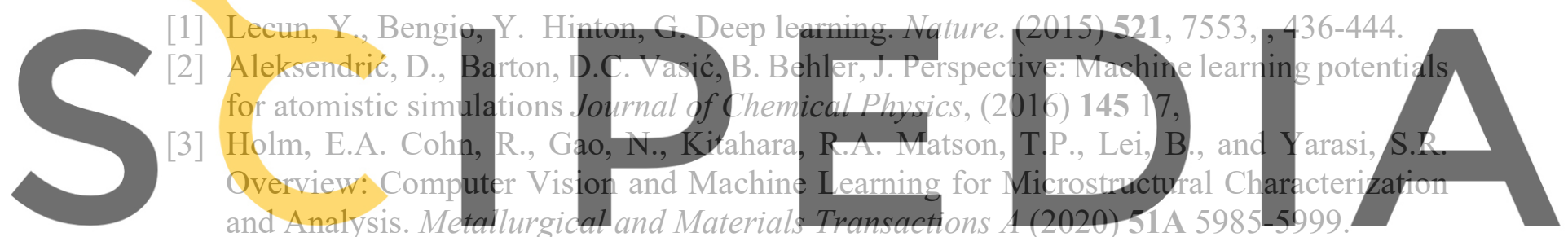

[4] Ashby M.F., Jones, D.R.H., Engineering Materials Volume 2: An Introduction to

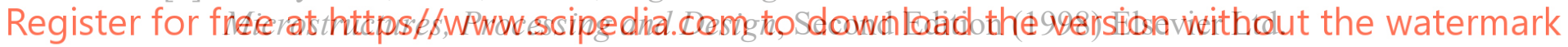

[5] Russ, J. Neal F.B., The Image Processing Handbook, 7th ed. (2016), CRC Press Taylor \&Francis Group

[6] Wang, Z.-L., Ogawa, T., Adachi, Y. Properties-to-microstructure-to-processing inverse analysis for steels via machine learning. ISIJ International. (2019) 59, 9, 1691-1694

[7] Vaško A. Evaluation of Shape of Graphite Particles in Cast Irons by a Shape Factor. Materials Today: Proceeding (2016) 34 1199-1204.

[8] Xiu, H., Ma F., Li, J., Zhao,X., Liu, L., Feng, P., Yang, X., Zhang, X., Kozliak, E., Ji, $Y$. Using fractal dimension and shape factors to characterize the microcrystalline cellulose (MCC) particle morphology and powder flowability Y. J.i., Powder Technology 2020, 364 241-250

[9] Brian L. DeCost, Elizabeth A. Holm, A. Computer vision approach for automated analysis and classification of microstructural image data. Comput. Mater. Sci. (2015) 110 126-133.

[10] Johnson, N.S., Vulimiri, P.S., To, A.C., Zhanga X., Bricea, C A., Kappesa, B., Stebner, P. Invited review: Machine learning for materials developments in metals additive manufacturing. Additive Manufacturing. (2020) 36101641.

[11] Chowdhury, A., Kautz, E., Yener, B., Lewis, D. Image driven machine learning methods for microstructure recognition, Comput. Mater.s Sci. (2016) 123 176-187. 
[12] Azimi, S.M., Britz, D., Engstler, M., Fritz, M., Mücklich, F. Advanced steel microstructural classification by deep learning methods. Scientific Reports ( 2018) 8, 1, 2128.

[13] Larmuseau, M, Sluydts, M. Theuwissen, K., Duprez, L., Dhaene, T, Cottenier, S. Race against the Machine: can deep learning recognize microstructures as well as the trained human eye? Scripta Materialia, (2021) 193 33-37.

[14] Xie, T., Grossman, J.C. Crystal Graph Convolutional Neural Networks for an Accurate and Interpretable Prediction of Material Properties (2018) Physical Review Letters, 120 (14), art. no. 145301

[15] Wang, Z.-L., Adachi, Y. Property prediction and properties-to-microstructure inverse analysis of steels by a machine-learning approach, Materials Science and Engineering A, (2019) 744 661-670.

[16]Ajioka,F. Wang, Z-L., OGAWA, T. and Adachi, Y. Development of High AccuracySegmentation Model for Microstructure of Steel by Deep Learning. ISIJ International. (2020), 60 5, 954-95.

[17]Hou, Y., Sapanathan, T., Dumon, A., Culière, P. Rachik, M. Novel artificial dual-phase microstructure generator based on topology optimization, Comput. Mater. Sci. (2016) 123 188-200.

[18] Krowiak, A. Radial basis function-based pseudospectral method for static analysis of thin plates. Engineering Analysis with Boundary Elements (2016) 71: 50-58.

[19]Krowiak. A. The application of the differential quadrature method based on a piecewise
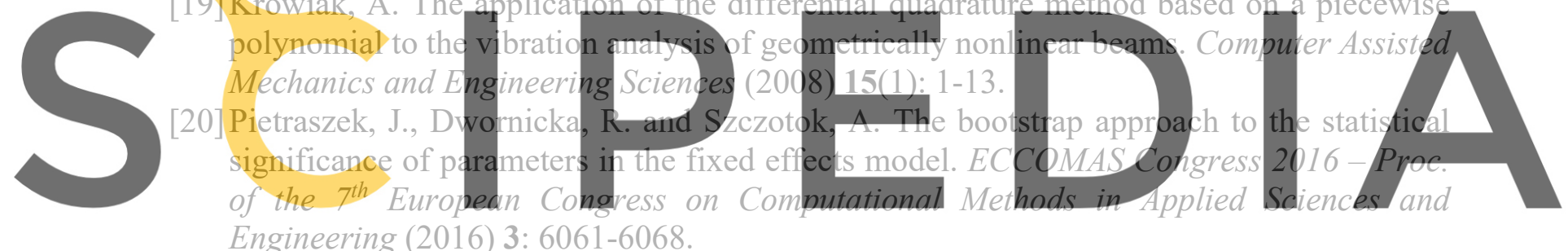

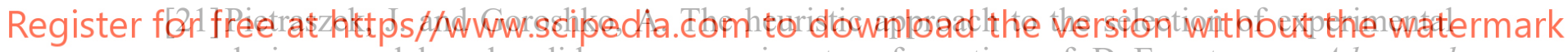
design, model and valid pre-processing transformation of DoE outcome. Advanced Materials Research (2014) 874: 145-149.

[22] Wojnar, L., Gadek-Moszczak, A. and Pietraszek, J. On the role of histomorphometric (stereological) microstructure parameters in the prediction of vertebrae compression strength. Image Analysis and Stereology (2019) 38(1): 63-73.

[23] Korzekwa, J., Gądek-Moszczak, A. and Zubko, M. Influence of the Size of Nanoparticles on the Microstructure of Oxide Coatings. Materials Science (2018) 53(5): 709-716.

[24] Bochenek, D., Niemiec, P., Korzekwa, J., Durtka, B. and Stokłosa, Z. Microstructure and properties of the ferroelectric-ferromagnetic PLZT-ferrite composites. Symmetry (2018) 10(3): art. 59.

[25] Korzekwa, J., Gądek-Moszczak, A. and Bara, M. The influence of sample preparation on SEM measurements of anodic oxide layers. Praktische Metallographie/Practical Metallography (2016) 53(1): 36-49.

[26]Dwornicka, R., Radek, N., Krawczyk, M., Osocha, P. and Pobędza, J. The laser textured surfaces of the silicon carbide analyzed with the bootstrapped tribology model. METAL 2017 - $26^{\text {th }}$ Int. Conf. on Metallurgy and Materials (2017): 1252-1257.

[27] Radek, N., Szczotok, A., Gądek-Moszczak, A., Dwornicka, R., Bronček, J. and 
Pietraszek, J. The impact of laser processing parameters on the properties of electro-spark deposited coatings. Archives of Metallurgy and Materials (2018) 63(2): 809-816.

[28]Pliszka, I. and Radek, N. Corrosion Resistance of WC-Cu Coatings Produced by Electrospark Deposition. Procedia Engineering (2017) 192: 707-712.

[29] Lipinski, T. and Karpisz, D. Effect of animal slurry on carbon structural S235JR steel at 303 K. Engineering for Rural Development (2020) 19: 1482-1487.

[30] Trzewiczek, K., Szczotok, A. and Gadek-Moszczak, A. Evaluation of the state for the material of the live steam superheater pipe coils of V degree. Advanced Materials Research (2014) 874: 35-42.

[31] Pacana, A., Czerwińska, K. and Dwornicka, R. Analysis of non-compliance for the cast of the industrial robot basis. METAL $2019-28^{\text {th }}$ Int. Conf. on Metallurgy and Materials (2019): 644-650.

[32] Karpisz, D. Design of manufacturing databases. Technical Transactions (2016) 113: 7377.

[33] Pietraszek, J., Sobczyk, A., Skrzypczak-Pietraszek, E. and Kołomycki, M. The fuzzy interpretation of the statistical test for irregular data. Technical Transactions (2016) 113: 119-126.

[34] Radek, N., Kurp, P. and Pietraszek, J. Laser forming of steel tubes. Technical Transactions (2019) 116: 223-229.

[35] Danielewski, H. Laser welding of pipe stubs made from super 304 steel. Numerical
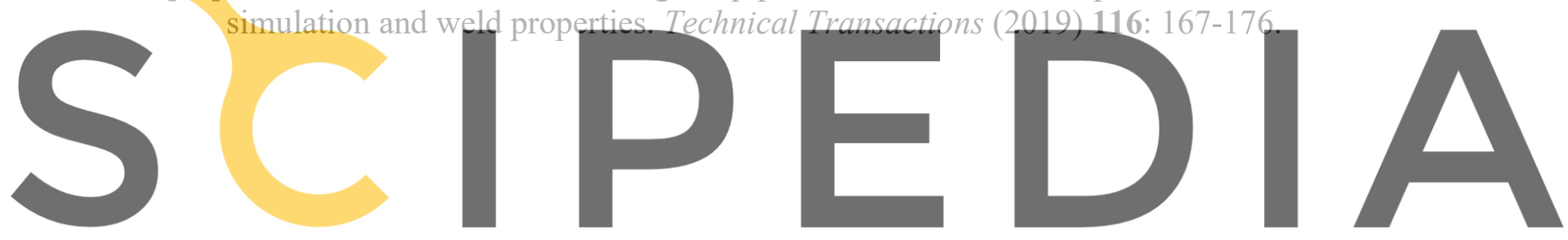

Register for free at https//www.scipedia.com to download the version without the watermark 